Article

\title{
Space-Time Characteristics of Vegetation Cover and Distribution: Case of the Henan Province in China
}

\section{Jingzhong Li ${ }^{1,2}$, Yongmei Liu ${ }^{1, *}$, Mingming Cao ${ }^{1}$ and Bing Xue ${ }^{3,4}$}

1 College of Urban and Environmental Science, Northwest University, Xi'an 710127, China; E-Mails: zhong_lij@163.com (J.L.); chengshi@nwu.edu.cn (M.C.)

2 College of Urban Planning and Landscape Garden, Xuchang University, Xuchang 461000, China

3 Lab for Urban \& Environmental Computation, Institute of Applied Ecology, Chinese Academy of Sciences, Shenyang 110016, China; E-Mail: xuebing@iae.ac.cn or bing.xue@iass-potsdam.de

4 Institute for Advanced Sustainability Studies (IASS), Potsdam 14467, Germany

* Author to whom correspondence should be addressed; E-Mail: liuym@nwu.edu.cn; Tel.: +86-29-8830-8412; Fax: +86-29-8830-8428.

Academic Editor: Marc A. Rosen

Received: 28 May 2015 / Accepted: 21 August 2015 / Published: 28 August 2015

\begin{abstract}
Vegetation indicators and spatial distribution characteristics are the core and basis to study the complex human-natural coupled system. In this paper, with Landsat 5 and Landsat 8 remote sensing data, we quantitatively estimated vegetation coverage in Henan Province, China. According to the urbanization rate, altitude, slope degree, and slope exposure, we analyzed spatial and temporal variation laws of vegetation coverage under the action of different factors to provide a reference for the improvement of the ecological environment and the quality assessment of Chinese granary. From 2000 to 2013, the vegetation coverage in Henan Province declined by $30.49 \%$ and the ecological environment deteriorated. The spatial change of vegetation coverage was evenly distributed in Henan Province. The vegetation coverage was increased in the west, south, and southwest parts of Henan Province and slightly decreased in the central, east, and the eastern part of Taihang Mountain. Vegetation coverage in a city was related to its population urbanization rate. The population urbanization rate was often negatively correlated with the vegetation coverage. According to the results of terrain factors based analysis, the low-altitude areas were in a good vegetation cover condition with the high vegetation coverage grade; the areas with a smaller slope degree had the large vegetation coverage and the coverage decreased with the increase in the slope degree; the coverage showed no significant difference between sunny and shady slopes and was less limited by light, temperature, and humidity.
\end{abstract}


Keywords: vegetation coverage; terrain factor; spatial and temporal variation; China

\section{Introduction}

With the rapid development of global economy and the increase in regional human activities, several aspects of natural ecosystems are gradually destroyed, especially in land utilization, ecological environment, and vegetation distribution [1-3]. As an important ecological factor for measuring natural ecosystem service, ground vegetation consisting of trees, shrubs, grass, and crops [4] has important influences on regional temperature and precipitation [5-7], and acts as the core and basis for establishing the sustainable human-natural system. Vegetation represents the overall condition of the regional ecological environment and its change process can not only reveal the environmental evolution [8], but also has positive feedback effects on the surface precipitation interception, soil and water conservation, and the improvement of the ecological environment [9].

Vegetation coverage is defined as the proportion of the vertical ground projection area of vegetation canopy in the total ground area [10]. It is one of the important measurement indicators of a regional ecological situation, and a key parameter indicating growth conditions and changes of surface plants. This parameter can be used to study vegetation changes within a given area, which is of major practical significance for understanding the current situation and development trend of the ecological environment in the area [11,12]. Remote sensing technology, which can synchronously acquire data information within a large area, has high precision and is slightly affected by subjective factors. Therefore, it has become the primary means for information retrieval of surface vegetation coverage and vegetation monitoring [13]. At present, Landsat 5 [14-18], Landsat 7 [18-20], Système Probatoire d'Observation de la Terre (SPOT) [21-23], and Moderate Resolution Imaging Spectroradiometer (MODIS) satellite [14,17,18,24-27] images are primarily used as the data sources for remote sensing monitoring on vegetation fractions. Images of Landsat 8 (the newly launched remote sensing satellite) were seldom used to investigate the variations in the vegetation fraction. In this study, with the Landsat 8 remote sensing data, the dimidiate pixel model was used to make a quantitative estimation of vegetation coverage in Henan Province. On the basis of the administrative map of Henan Province and terrain factors extracted by digital elevation model (DEM), spatial variations of vegetation coverage in Henan Province were explored. Historical data were used to analyze the inter-annual variability of vegetation coverage in Henan Province so as to provide an essential basis for urban development and ecological environment improvement in Henan Province.

Henan Province, a great agricultural province, has long been playing a crucial role in ensuring national food security and promoting economic and social development. However, in recent years, due to the rapid development and formation of the central Henan Urban Agglomeration, partial areas have suffered vegetation degradation, soil erosion, and ecological environment deterioration, which have greatly affected the ecological balance and sustainable development of the economy. However, as an important indicator, vegetation coverage can be used to study spatial and temporal variations of ground vegetation, explore the influences of social activities on the environment, and effectively monitor the ecosystem status [28]. Therefore, effectively monitoring the spatial and temporal development status of 
vegetation in Henan Province is of decisive significance for reducing the deterioration of the ecological environment caused by the changes in vegetation coverage and the output fluctuation of agricultural and sideline products.

\section{Methods and Data}

\subsection{Study Area}

Henan Province is located in mid-eastern China and the middle and lower reaches of the Yellow River $\left(31^{\circ} 23^{\prime} \mathrm{N} \sim 36^{\circ} 22^{\prime} \mathrm{N}, 110^{\circ} 21^{\prime} \mathrm{E} \sim 116^{\circ} 39^{\prime} \mathrm{E}\right.$ ) and covers an area of about 167,000 $\mathrm{km}^{2}$ (Figure 1). From the geographical perspective, the central and eastern areas of Henan Province are located in the south of the North China Plain, while the western and southern areas are located in the Nanyang Basin. Henan Province belongs to the warm temperate subtropical zone and has a humid-subhumid monsoon climate. The annual average temperature in Henan Province ranges from 12 to $16^{\circ} \mathrm{C}$. In Henan Province, it is usually cold in the winter and spring with less rainfall, but hot in the summer and autumn with more rainfall and sufficient sunshine time. The average annual precipitation is about $500-900 \mathrm{~mm}$. However, half of the precipitation occurs in the summer. Soils in Henan Province are mainly composed of natural soils (brown soil, cinnamon soil, yellow brown soil, and yellow cinnamon soil) and agricultural soils (alluvial soil and mortar black soil). Vegetation is mainly the broad-leaved deciduous forest in the warm temperate zone and the evergreen broad-leaved deciduous forest in the northern subtropical zone. The area of mountains and hills in Henan Province is $74,000 \mathrm{~km}^{2}$, accounting for $44.3 \%$ of the total area of Henan Province, while the area of plains and basins is $93,000 \mathrm{~km}^{2}$, accounting for $55.7 \%$ of the total area. Complex and diverse lands provide favorable conditions for the comprehensive development of forestry, farming animal husbandry, and fisheries in Henan Province.

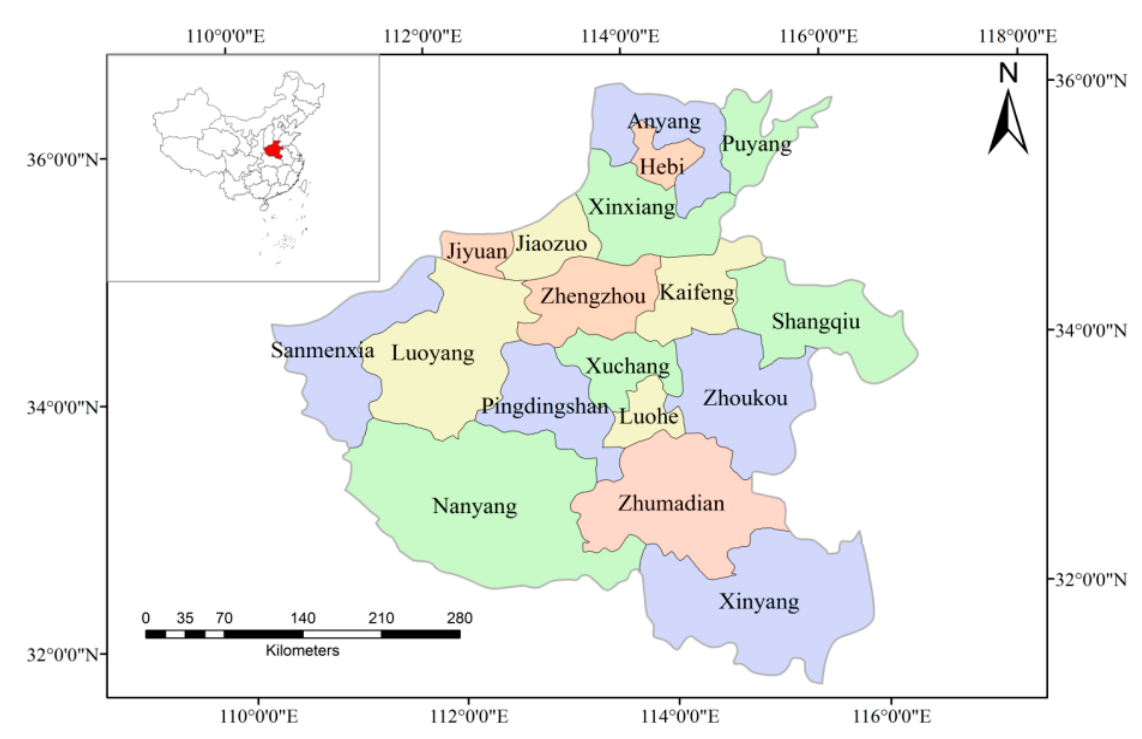

Figure 1. Location of the study area.

\subsection{Data Sources and Pre-Processing}

In this paper, DEM of Henan Province (ASTER GDEM, spatial resolution of $30 \mathrm{~m}$ ), the administrative map of Henan Province, TM images, the Statistical Yearbook of Henan, and Landsat 8 data images from 
websites of the Geographic Data Service Center of the United States Geological Survey (USGS) and The Center for Earth Observation and Digital Earth, Chinese Academy of Sciences, were used. Remote sensing data used in this paper are mainly the data images (14 images) of vegetation cover in July and August from 2000 to 2013. In July and August, vegetation is usually in a suitable growth condition, so that vegetation can be easily differentiated from soil. Under these conditions, it is convenient to extract effective data about vegetation coverage. Moreover, radiometric calibration, Fast Line-of-Sight Atmospheric Analysis of Spectral Hypercubes (FLAASH) atmospheric correction, seamless mosaics, cropping, and other pre-processing steps were performed with collected remote sensing images to acquire basic remote sensing images of the study area.

\subsection{Extraction of Vegetation Coverage}

Vegetation index, as a parameter indicating vegetation information with different combinations of red and infrared bands, can be used to quantitatively reflect the vegetation vitality. Compared with single bands, this parameter shows a higher sensitivity in measuring the vegetation biomass [29]. Currently, 150 models on vegetation indices are available in the world. However, only a few models have been systematically validated and applied [30]. With the high detection sensitivity and wide detection range, the Normalized Difference Vegetation Index (NDVI) can be used to eliminate shadows and radiated interference from terrains and community structures, and to weaken noise caused by solar elevation angle and atmosphere. Moreover, as NDVI is effective for assessing vegetation situations, it has been widely used in the surface vegetation productivity assessment, vegetation cover estimation, and variation trend analysis [31-33]. In this study, NDVI is used and calculated by:

$$
\mathrm{NDVI}=(\mathrm{NIR}-\mathrm{R}) /(\mathrm{NIR}+\mathrm{R})
$$

where NIR denotes the near-infrared bands and $\mathrm{R}$ is the red bands [34].

In this paper, NDVI was used as the main parameter in a dimidiate pixel model to calculate vegetation coverage in Henan Province. According to the basic calculation principle, a pixel NDVI value can be expressed as the sum of the information contributed by vegetation-covered areas and the information contributed by non-vegetation-covered (bare soil) areas. Vegetation Fractional Coverage (VFC) in the dimidiate pixel model is calculated as:

$$
\mathrm{VFC}=\left(\mathrm{NDVI}-\mathrm{NDVI}_{\min }\right) /\left(\mathrm{NDVI} \max -\mathrm{NDVI}_{\min }\right)
$$

where NDVI $I_{\max }$ and NDVI min are respectively the maximum and minimum NDVI values in an area. Due

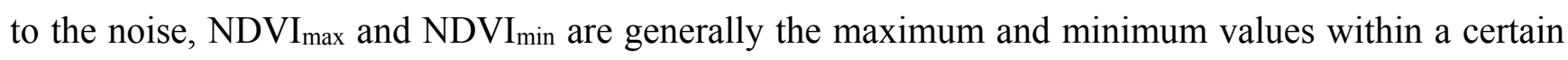
confidence interval, which are also pure vegetation pixel and pure soil pixel. Due to the impact of vegetation distribution and the radiation of surrounding surface features, the values of confidence coefficients are mainly determined by the actual situation of images in the study area. In the case that a large number of measured data are not available for reference, the cumulative percentages (5\% and 95\%) are usually taken as confidence intervals [35]. In this paper, $5 \%$ is taken as the pure pixel of bare soil and $95 \%$ is taken as the pure vegetation pixel. Vegetation cover images are calculated by Equation (2). 


\subsection{Grade Classification of Vegetation Coverage}

On the basis of the vegetation coverage images and the standards by Chen [36] and Ma [37] for the grading of vegetation coverage, vegetation coverage was divided into five grades (see Table 1). Vegetation density segmentation was performed according to the grading standards. The areas with the same vegetation density range are printed with the same color. The decision tree method was adopted to generate the vegetation coverage grading figure.

Table 1. Vegetation coverage grade classification.

\begin{tabular}{cccccc}
\hline $\begin{array}{c}\text { Vegetation Fractional } \\
\text { Coverage/(\%) }\end{array}$ & VFC $<\mathbf{2 0}$ & $\mathbf{2 0} \leq \mathrm{VFC}<\mathbf{4 0}$ & $\mathbf{4 0} \leq \mathrm{VFC}<\mathbf{6 0}$ & $\mathbf{6 0} \leq \mathrm{VFC}<\mathbf{8 0}$ & $\mathbf{8 0} \leq \mathrm{VFC}$ \\
\hline Grades & Extremely low & Low & Medium & Upper medium & High \\
\hline
\end{tabular}

\section{Results and Discussion}

\subsection{Inter-Annual Variation of Vegetation Coverage}

ENvironment for Visualizing Images (ENVI) can be used as a statistical tool to respectively calculate the annual average vegetation coverage in Henan Province in 2000 (0.455939) and 2013 (0.316906). It can be seen that the annual average vegetation coverage in 2013 is $30.49 \%$ lower than that in 2000 , showing a downward trend. From 2000 to 2013, from the vegetation coverage grade variation figure (Figure 2) in Henan Province and the vegetation coverage variation percentages in the cities of Henan Province (Figure 3), it can be seen that average vegetation coverage variation percentages of Zhumadian, Luoyang, Xinyang, and Nanyang were respectively 1.17\%, 1.35\%, 1.51\%, and 1.91\%. These four cities experienced a significant increase in their vegetation coverage percentages. Among them, Nanyang and Xinyang showed the most significant increase. Vegetation coverage was improved in Sanmenxia, Zhoukou, and Shangqiu, which respectively showed different increase percentages of $0.92 \%, 0.72 \%$, and $0.78 \%$. However, in northern areas of Nanyang, near Funiu Mountain and Tongbai Mountain, the vegetation coverage of partial areas in Zhumadian and Zhoukou and northern areas in Xinxiang showed a significant decreasing trend.

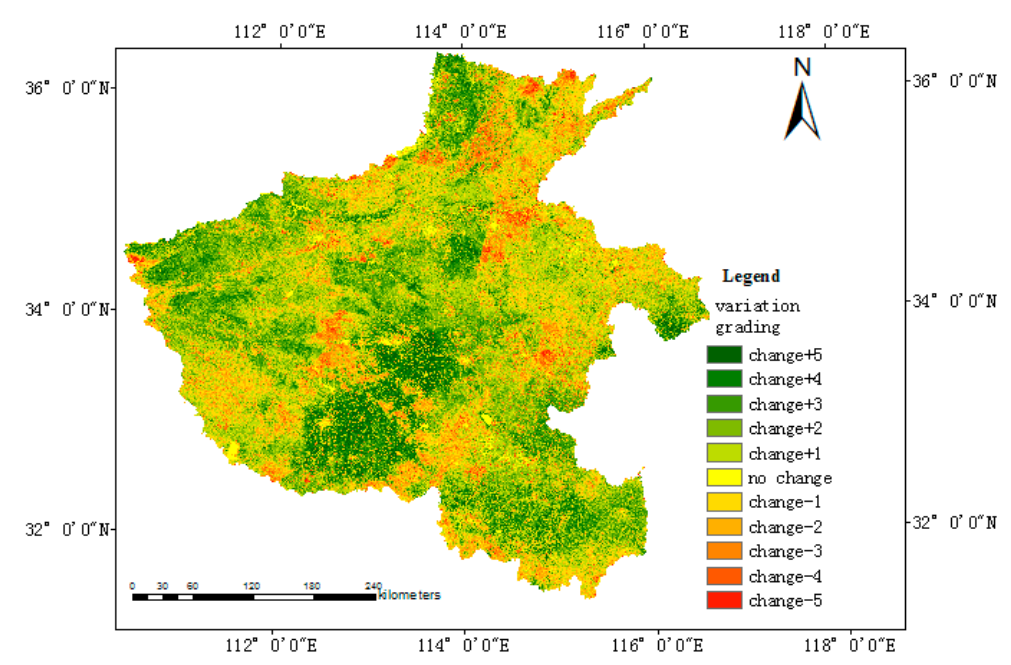

Figure 2. Vegetation coverage variation grading in Henan Province. 


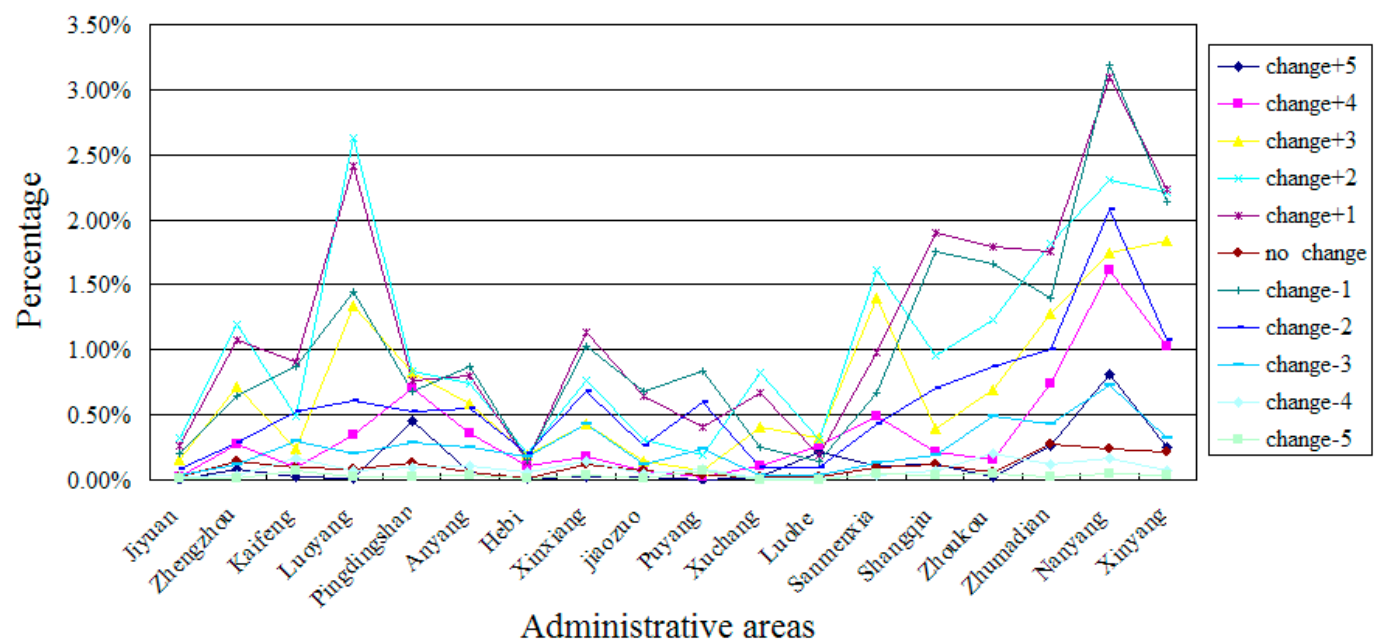

Figure 3. Vegetation coverage variation percentages in different administrative areas of Henan Province.

\subsection{Spatial Variations of Vegetation Coverage in Different Cities in Henan Province}

Based on an analysis of statistical data of spatial variations of vegetation coverage in different cities in Henan Province in 2013 (Figure 4), it can be seen that Luohe, Shangqiu, Xuchang, Zhumadian, and Sanmenxia, respectively, have the higher average vegetation coverage of $0.704,0.699,0.679,0.649$, and 0.634. However, the average vegetation coverage of Zhengzhou (0.506) was the lowest. According to an exploration of the relationship between the average vegetation coverage of a city and its urbanization level [38], it could be concluded that a higher average vegetation coverage of a city indicates a lower urbanization rate of the city. Luohe, Shangqiu, Xuchang, Zhumadian, and partial areas of Sanmenxia had a relatively higher vegetation coverage. The urbanization process in these cities and areas was relatively slow, so the influences of human activities in these cities and areas were quite small. As a result, the average vegetation coverage in those cities was relatively higher. Conversely, in the cities such as Zhengzhou, Pingdingshan, and Jiyuan, with a higher urbanization rate and well-developed economy, average vegetation coverage was much lower.

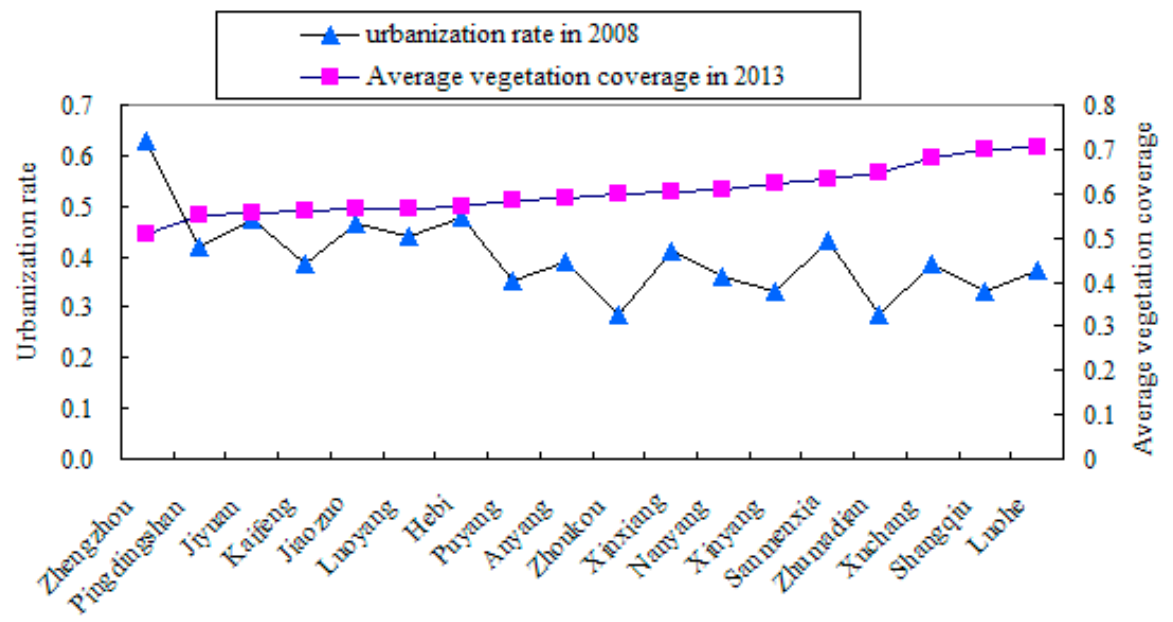

Figure 4. Contrastive analysis of the relationship between average vegetation coverage and urbanization rate of each city in Henan Province. 


\subsection{Vegetation Coverage Variations Caused by Terrain Factors}

Terrain factors, mainly including elevation, slope degree, and slope exposure, to a certain extent, affect human activities. In particular, the changes in surface light, heat, temperature, and other natural conditions would result in the transfer and redistribution of water, heat, and nutrients and indirectly affect vegetation growth and development. In this paper, DEM data of Henan Province were used to extract information about terrain factors including altitude, slope degree, and slope exposure. On this basis, spatial distribution variations of vegetation coverage in Henan Province were analyzed and assessed from the perspective of the three terrain factors.

\subsubsection{Spatial Variations of Vegetation Coverage in Different Altitudes}

According to DEM data, sunshine, temperature, precipitation, and other climate factors in Henan Province $[39,40]$, the areas of Henan Province can be divided into eight altitude groups: $<200 \mathrm{~m}$, 200-600 m, 600-1000 m, 1000-1400 m, 1400-1800 m, 1800-2200 m, 2200-2600 m, and >2600 m. After combining each altitude group with corresponding vegetation coverage from 2013, the vegetation coverage distribution in different altitude groups was plotted (see Figure 5). The medium-altitude areas (600-1000 m, 1000-1400 m, and 1400-1800 m) had the higher average vegetation coverage $(0.65,0.72$, and 0.709$)$. The average vegetation coverage of low-altitude areas $(<200 \mathrm{~m}$ and 200-600 $\mathrm{m}$ ) was lower than 0.605 . In particular, the average vegetation coverage of the areas in altitude groups of 200-600 m dropped significantly because there was a clear indication of human activities in low-altitude areas ( $<200 \mathrm{~m}$ and 200-600 m). Urban construction, resource extraction, and other activities greatly changed the ecological environment. However, in the high-altitude areas (1800-2200 m, 2200-2600 m, and $>2600 \mathrm{~m}$ ), due to the impact of heat, moisture, soil fertility, and other factors, the vegetation coverage decreased significantly. Vegetation in high-altitude areas is mainly composed of alpine vegetation, such as broad-leaved mingled forests, mountain cold-temperate coniferous forests, bush fallow, and meadow. As shown in Figure 6, with the increase in altitudes, vegetation coverage of all grades showed a significant decreasing trend. In particular, the areas with the altitude of 200-600 m experienced the sharpest decrease. Vegetation coverage of all grades dropped sharply mainly due to the great impact of human activities. However, with the further increase in altitudes, human interference of vegetation coverage of each grade was reduced. Therefore, the decreasing trend of vegetation coverage became less significant. In the high-altitude areas, the vegetation cover of each grade was gradually reduced to zero. The average vegetation coverage of high-mountain areas was 0.426 . However, high-mountain areas just accounted for a small proportion in the study area.

On the whole, although low-altitude areas were greatly affected by human activities, low-altitude areas still had relatively high vegetation coverage. The overall vegetation coverage in low-altitude areas was $68.51 \%$. Moreover, its coverage grade was very high. With an increase in the altitude, the average vegetation coverage increased. However, limited by vegetation growth conditions, vegetation cover areas in each coverage grade was significantly reduced. In particular, in high-altitude areas, the vegetation coverage was close to zero. 


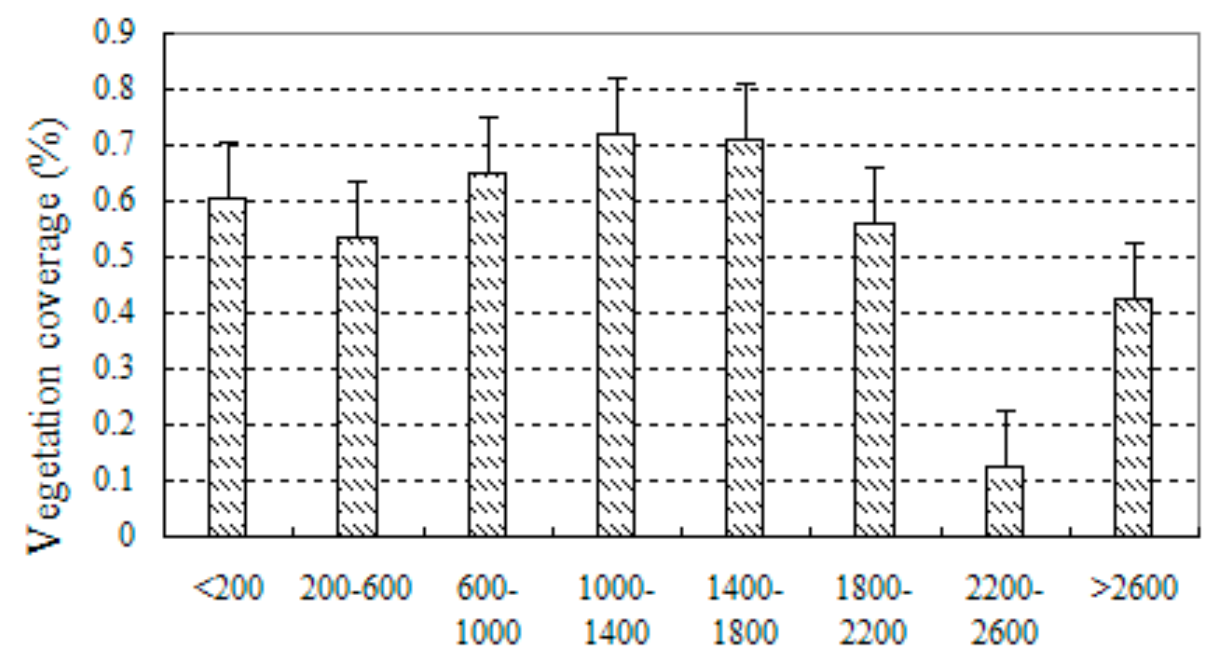

Altitude group (m)

Figure 5. Vegetation coverage distributions in different altitude groups.

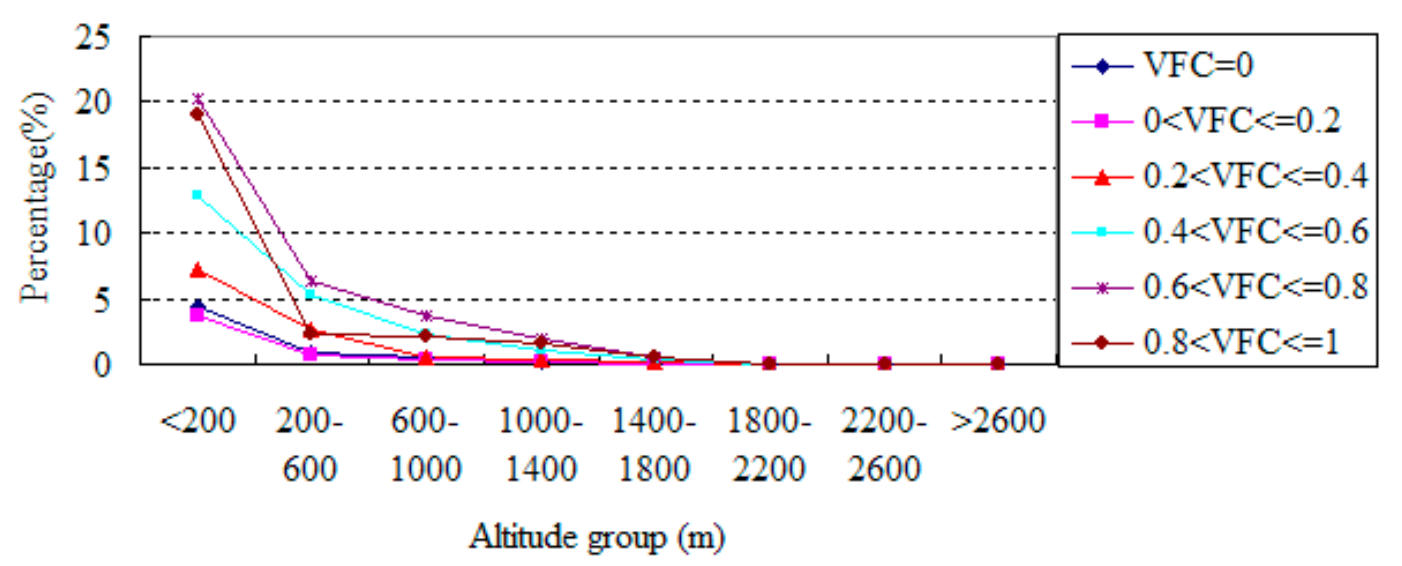

Figure 6. Vegetation coverage percentages of different altitude groups.

\subsubsection{Spatial Variations of Vegetation Coverage in Different Slope Degrees}

Information about slope degrees was extracted from DEM data of the study area. In terms of slope degrees, the areas in Henan Province were divided into seven groups: $0-5^{\circ}, 5-10^{\circ}, 10-20^{\circ}, 20-30^{\circ}$, $30-40^{\circ}, 40-50^{\circ}$, and $>50^{\circ}$. Combining the area of each slope degree with corresponding vegetation coverage from 2013, the vegetation coverage percentage in each slope degree group was obtained (see Table 2). From Table 2 and Figure 7, it can be seen that in small slope degree areas, vegetation cover areas were relatively large and mainly composed of medium and high vegetation coverage. In particular, in the areas of the $0-5^{\circ}$ slope degree group, vegetation cover areas accounted for $67.42 \%$ of the total area. As the data were harvested in summer, high-coverage vegetation was mainly crops. With the increase in the slope degree, terrain became increasingly steep. Percentages of vegetation cover areas gradually dropped. In large-slope-degree areas, human activities were less frequent. Woodland was the main vegetation in this area. 
Table 2. Vegetation cover percentages in different slope degree groups (\%).

\begin{tabular}{cllllllll}
\hline Slope Degrees $\left({ }^{\circ}\right)$ & $\mathbf{0}-\mathbf{5}$ & $\mathbf{5}-\mathbf{1 0}$ & $\mathbf{1 0}-\mathbf{2 0}$ & $\mathbf{2 0}-\mathbf{3 0}$ & $\mathbf{3 0}-\mathbf{4 0}$ & $\mathbf{4 0}-\mathbf{5 0}$ & $>\mathbf{5 0}$ & Total \\
\hline $\mathrm{FC}=0$ & 4.37 & 0.86 & 0.53 & 0.21 & 0.07 & 0.01 & 0.00 & 6.06 \\
$0<\mathrm{FC} \leq 0.2$ & 3.95 & 0.67 & 0.30 & 0.10 & 0.03 & 0.01 & 0.00 & 5.07 \\
$0.2<\mathrm{FC} \leq 0.4$ & 7.87 & 1.66 & 1.04 & 0.41 & 0.13 & 0.02 & 0.00 & 11.13 \\
$0.4<\mathrm{FC} \leq 0.6$ & 12.83 & 3.29 & 2.62 & 1.12 & 0.33 & 0.04 & 0.00 & 20.25 \\
$0.6<\mathrm{FC} \leq 0.8$ & 20.19 & 4.66 & 4.07 & 1.95 & 0.53 & 0.06 & 0.01 & 31.48 \\
$0.8<\mathrm{FC} \leq 1$ & 18.20 & 2.92 & 2.58 & 1.67 & 0.55 & 0.08 & 0.01 & 26.01 \\
Total & 67.42 & 14.07 & 11.15 & 5.47 & 1.65 & 0.23 & 0.03 & 100.00 \\
\hline
\end{tabular}

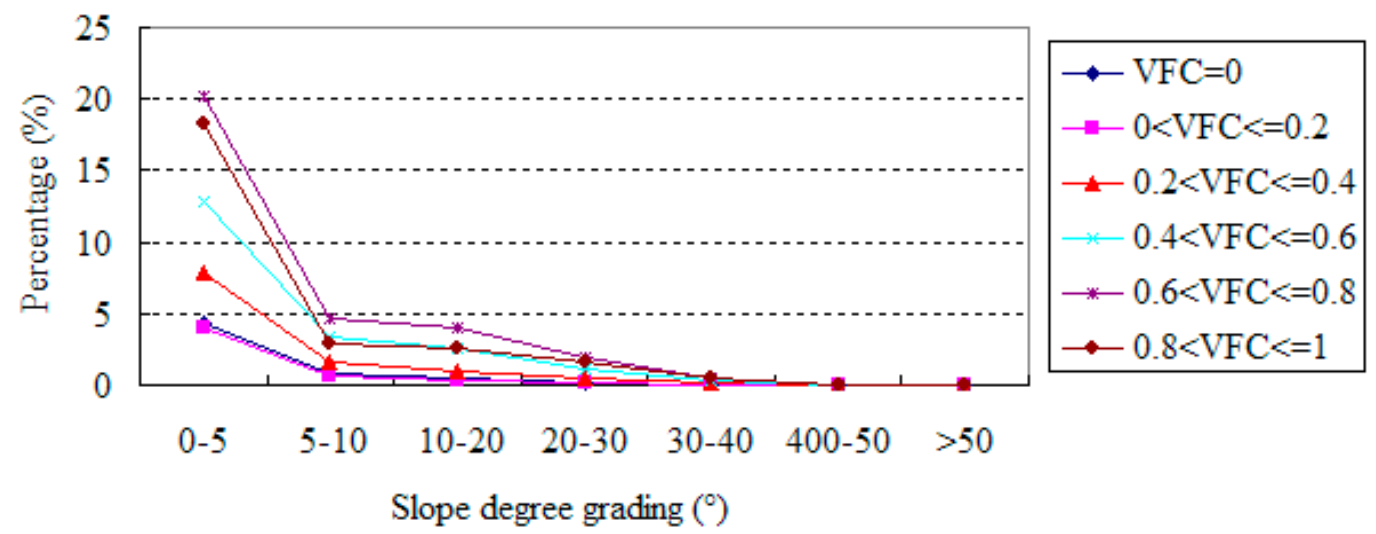

Figure 7. Vegetation cover percentages in different slope degree groups.

\subsubsection{Spatial Variations of Vegetation Coverage in Different Slope Exposures}

Information about slope exposures was extracted based on DEM data of Henan Province. In terms of slope exposures, the areas in Henan Province are divided into eight groups: $0-45^{\circ}, 45-90^{\circ}, 90-135^{\circ}$, $135-180^{\circ}, 180-225^{\circ}, 225-270^{\circ}, 270-315^{\circ}$, and $315-360^{\circ}$. On the basis of vegetation coverage from 2013, vegetation coverage percentages of different slope exposure groups were calculated. According to Table 3 and Figure 8, vegetation cover areas of the $0-45^{\circ}$ slope exposure group were the largest. Vegetation coverage percentages in other slope exposure groups did not greatly differ from each other. According to the statistics of sunny and shady slopes, vegetation cover areas of sunny slopes and shady slopes respectively accounted for $50.61 \%$ and $49.39 \%$ of the total vegetation cover area. Vegetation coverage of sunny slopes was slightly higher than that of shady slopes. Taking data acquisition time into account, the impact of slope exposure on the study area was quite small in summer. Vegetation coverage in different slope exposures slightly differed from each other and were less limited by light, temperature, and humidity. 
Table 3. Vegetation cover percentages in different slope exposure groups (\%).

\begin{tabular}{cccccccccc}
\hline Slope Exposure $\left(^{\circ}\right)$ & $\mathbf{0 - 4 5}$ & $\mathbf{4 5}-\mathbf{9 0}$ & $\mathbf{9 0}-\mathbf{1 3 5}$ & $\mathbf{1 3 5}-\mathbf{1 8 0}$ & $\mathbf{1 8 0}-\mathbf{2 2 5}$ & $\mathbf{2 2 5}-\mathbf{2 7 0}$ & $\mathbf{2 7 0}-\mathbf{3 1 5}$ & $\mathbf{3 1 5}-\mathbf{3 6 0}$ & Total \\
\hline $\mathrm{VFC}=0$ & 1.09 & 0.70 & 0.77 & 0.77 & 0.77 & 0.71 & 0.71 & 0.54 & 6.06 \\
$0<\mathrm{VFC} \leq 0.2$ & 0.82 & 0.60 & 0.66 & 0.69 & 0.70 & 0.60 & 0.57 & 0.42 & 5.07 \\
$0.2<\mathrm{VFC} \leq 0.4$ & 1.77 & 1.33 & 1.43 & 1.47 & 1.50 & 1.33 & 1.30 & 1.00 & 11.13 \\
$0.4<\mathrm{VFC} \leq 0.6$ & 3.29 & 2.37 & 2.43 & 2.44 & 2.58 & 2.50 & 2.57 & 2.07 & 20.25 \\
$0.6<\mathrm{VFC} \leq 0.8$ & 5.26 & 3.88 & 3.91 & 3.79 & 3.95 & 3.77 & 3.83 & 3.08 & 31.48 \\
$0.8<\mathrm{VFC} \leq 1$ & 4.36 & 3.44 & 3.76 & 3.48 & 3.20 & 2.81 & 2.82 & 2.16 & 26.01 \\
Total & 16.58 & 12.32 & 12.96 & 12.64 & 12.69 & 11.73 & 11.80 & 9.28 & 100.00 \\
\hline
\end{tabular}

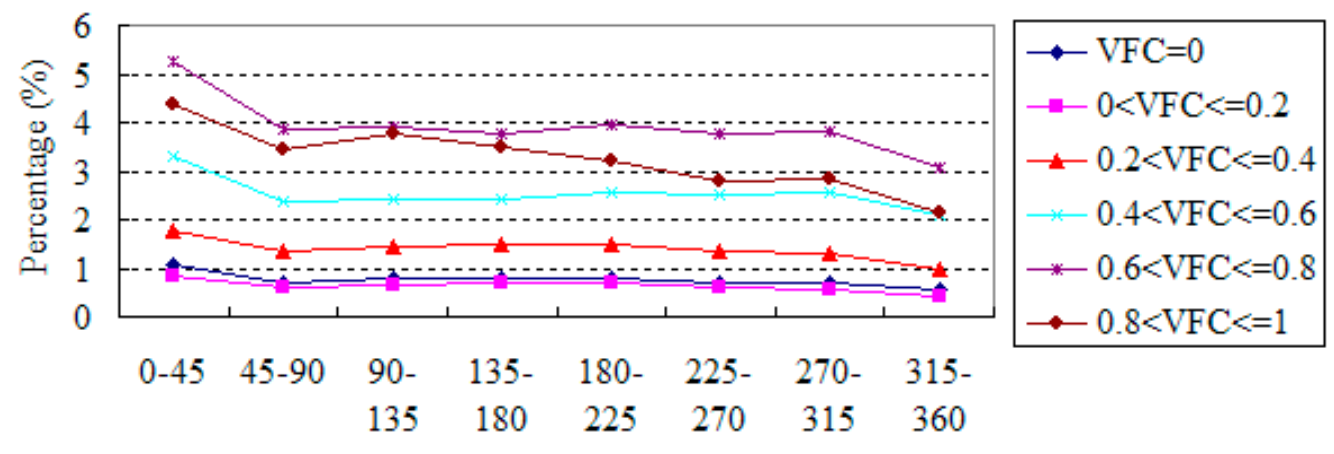

Slop exposure groups $\left({ }^{\circ}\right)$

Figure 8. Vegetation coverage percentages of different slope exposure groups.

\section{Conclusions}

Based on the remote sensing data from Landsat 5 in 2000 and Landsat 8 in 2013, vegetation coverage in different periods in Henan Province was extracted. According to the administrative map of Henan Province and its terrain factors, we analyzed the spatial and temporal differences in vegetation coverage under the action of different factors and obtained the following conclusions. From 2000 to 2013, the vegetation coverage in Henan Province decreased by $30.49 \%$ and the ecological environment deteriorated. The spatial change of vegetation coverage was relatively evenly distributed in Henan Province. The vegetation coverage was increased in Luoyang, Sanmenxia, Nanyang, and Xinyang in the west of Henan Province and slightly decreased in Zhumadian, Zhoukou, and the eastern part of Taihang Mountain in Henan Province. Vegetation coverage in a city was related to its population urbanization rate. The areas with the high vegetation coverage were mainly Shangqiu, Zhumadian, Sanmenxia, and other regions with a low population urbanization rate and slow urbanization process. The areas with the low vegetation coverage were mainly Zhengzhou, Pingdingshan, Jiyuan, and other regions with a rapid urbanization process and high economic development level. According to the analysis results of terrain factors, low-altitude areas in Henan Province were in good vegetation coverage condition and the overall vegetation coverage in low-altitude areas was $68.51 \%$. Average vegetation coverage increased with the increase in the altitude. Limited by vegetation growth conditions, both vegetation coverage areas at all grades and total vegetation cover areas were decreased. In small slope degree areas, vegetation cover areas were relatively large and mainly composed of medium-level and high-level vegetation coverage. With the increase in the slope degree, terrain became increasingly steep and the percentages of vegetation cover area of all grades gradually dropped. According to the analysis of slope exposure 
conditions, the coverage showed no significant difference between sunny and shady slopes and was less limited by light, temperature, and humidity.

\section{Acknowledgments}

The study was supported by the National Science Foundation of China (Nos. 41171225, 41471116) and the Program for Soft Technology Study Plan in Henan Province (No. 132400411021).

\section{Author Contributions}

Jingzhong Li contributed to data collection, data processing, and drafting the manuscript. Yongmei Liu conducted the research, the structural design, data analysis, and paper revision. Mingming Cao and Bing Xue contributed to data analysis and paper revision. All authors have read and approved the final manuscript.

\section{Conflicts of Interest}

The authors declare no conflict of interest.

\section{References}

1. Chen, X.; Li, B.L. Effect of global climate change and human disturbances on tree diversity of the forest regenerating from clear-cuts of mixed broadleaved korean pine forest in Northeast China. Chemosphere 2003, 51, 215-226.

2. Bucała, A. The impact of human activities on land use and land cover changes and environmental processes in the Gorce Mountains in the past 50 years. J. Environ. Manag. 2014, 138, 4-14.

3. Hunt, C.O.; Premathilake, R. Early Holocene vegetation, human activity and climate from Sarawak Malaysian Borneo. Quat. Int. 2012, 249, 105-119.

4. Zhang, Y.; Yang, J.; Wu, H.; Shi, C.; Zhang, C.; Li, D.; Feng, M. Dynamic changes in soil and vegetation during varying ecological recovery conditions of abandoned mines in Beijing. Ecol. Eng. 2014, 73, 676-683.

5. Defnies, R.S.; Townshend, J.R.G. NDVI-derived land cover classifications at a global scale. Int. J. Remote Sens. 1994, 17, 3567-3586.

6. Perini, K.; Magliocco, A. Effects of vegetation, urban density, building height, and atmospheric conditions on local temperatures and thermal comfort. Urban For. Urban Green. 2014, 13, 495-506.

7. Fan, Z.M.; Li, J.; Yue, T.X. Changes of Climate-Vegetation Ecosystem in Loess Plateau of China. Procedia Environ. Sci. 2012, 13, 715-720.

8. Liu, X.; Zhang, W.; Yang, F.; Zhou, X.; Liu, Z.; Qu, F.; Lian, S.; Wang, C.; Tang, X. Changes in vegetation-environment relationships over long-term natural restoration process in Middle Taihang Mountain of North China. Ecol. Eng. 2012, 49, 193-200.

9. Zhou, Z.C.; Shangguan, Z.P.; Zhao, D. Modeling vegetation coverage and soil erosion in the Loess Plateau Area of China. Ecol. Model. 2006, 198, 263-268.

10. Purevdoij, T.; Tateishi, R.; Ishiyama, T.; Honda, Y. Relationships between percent vegetation cover and vegetation indices. Int. J. Remote Sens. 1998, 18, 3519-3535. 
11. Gong, Z.; Cui, T.; Pu, R.; Lin, C.; Chen, Y. Dynamic simulation of vegetation abundance in a reservoir riparian zone using a sub-pixel Markov model. Int. J. Appl. Earth Obs. Geoinform. 2015, $35,175-186$.

12. Leprieur, C.; Mastorchio, S.; Meunier, J.C.; Kerr, Y.H. Monitoring vegetation cover across semi-arid regions: Comparison of remote observations from various scales. Int. J. Remote Sens. 2000, 2, 281-300.

13. Harris, A.; Carr, A.S.; Dash, J. Remote sensing of vegetation cover dynamics and resilience across southern Africa. Int. J. Appl. Earth Obs. Geoinform. 2014, 28, 131-139.

14. Jiapaer, G.; Chen, X.; Bao, A. A comparison of methods for estimating fractional vegetation cover in arid regions. Agric. For. Meteorol. 2011, 151, 1698-1710.

15. Jing, X.; Yao, W.Q.; Wang, J.H.; Song, X.Y. A study on the relationship between dynamic change of vegetation coverage and precipitation in Beijing's mountainous areas during the last 20 years. Math. Comput. Model. 2011, 54, 1079-1085.

16. Adams, M.P.; Smith, P.L. A systematic approach to model the influence of the type and density of vegetation cover on urban heat using remote sensing. Landsc. Urban Plan. 2014, 132, 47-54.

17. Yang, J.; Weishberg, P.J.; Bristow, N.A. Landsat remote sensing approaches for monitoring long-term tree cover dynamics in semi-arid woodlands: Comparison of vegetation indices and spectral mixture analysis. Remote Sens. Environ. 2012, 119, 62-71.

18. Jia, K.; Liang, S.; Zhang, L.; Wei, X.; Yao, Y.; Xie, X. Forest cover classification using landsat ETM+ data and time series MODIS NDVI data. Int. J. Appl. Earth Obs. Geoinform. 2014, 33, $32-38$.

19. Salovaara, K.J.; Thessler, S.; Malik, R.N.; Tuomisto, H. Classification of Amazonian primary rain forest vegetation using Landsat ETM+ satellite imagery. Remote Sens. Environ. 2005, 97, 39-51.

20. Hais, M.; Jonasova, M.; Langhammer, J.; Kucera, T. Comparison of two types of forest disturbance using multitemporal landsat TM/ETM+ imagery and field vegetation data. Remote Sens. Environ. 2009, 113, 835-845.

21. Eckert, S.; Engesser, M. Assessing vegetation cover and biomass in restored erosion areas in Iceland using SPOT satellite data. Appl. Geogr. 2013, 40, 179-190.

22. Jarlan, L.; Mangiarotti, S.; Mougin, E.; Mazzega, P.; Hiernaux, P.; Dantec, V.L. Assimilation of SPOT/VEGETATION NDVI data into a sahelian vegetation dynamics model. Remote Sens. Environ. 2008, 112, 1381-1394.

23. Dymond, J.R.; Stephens, P.R.; Newsome, P.F.; Wilde, R.H. Percentage vegetation cover of a degrading rangeland from SPOT. Int. J. Remote Sens. 1992, 11, 1999-2007.

24. Wardlow, B.D.; Egbert, S.L.; Kastens, J.H. Analysis of time-series MODIS $250 \mathrm{~m}$ vegetation index data for crop classification in the U.S. Central Great Plains. Remote Sens. Environ. 2007, 108, 290-310.

25. Li, Z.; Li, X.; Wei, D.; Xu, X.; Wang, H. An assessment of correlation on MODIS-NDVI and EVI with natural vegetation coverage in Northern Hebei Province China. Procedia Environ. Sci. 2010, 2, 946-969.

26. Bajocco, S.; Angelis, A.D.; Salvati, L. A satellite-based green index as a proxy for vegetation cover quality in a Mediterranean region. Ecol. Indic. 2012, 23, 578-587. 
27. Zhang, J.; Liu, Z.; Sun, X. Changing landscape in the Three Gorges Reservoir Area of Yangtze River from 1977 to 2005: Land use/land cover, vegetation cover changes estimated using mult-source satellite data. Int. J. Appl. Earth Obs. Geoinform. 2009, 11, 403-412.

28. Xin, Z.; Xu, J.; Zheng, W. Impact of climatic changes and human activities on vegetation coverage changes in the Loess Plateau. Sci. China (Ser. D) Earth Sci. 2007, 11, 1504-1514. (In Chinese)

29. Baret, F.; Guyot, G.; Major, D.J. TSAVI: A vegetation index which minimizes soil brightness effects on LAI and APPR estimation. In Proceedings of the 12th Canadian Symposium on Remote Sensing, Vancouver, BC, Canada, 10-14 July 1989; pp. 1355-1358.

30. Deng, S.; Chen, Q. Overview of vegetation spectral characteristics and vegetation index. In Proceedings of the 17th China Symposium on Remote Sensing, Hangzhou, China, 27-31 August 2010. (In Chinese)

31. Paruelo, J.M.; Epstein, H.E.; Lauenroth, W.K.; Burke, I.C. ANPP estimates from NDVI for the central grassland region of the United States. Ecology 1997, 3, 953-958.

32. Fensholt, R.; Proud, S.R. Evaluation of Earth Observation based global long term vegetation trends-Comparing GIMMS and MODIS global NDVI time series. Remote Sens. Environ. 2012, 119, 131-147.

33. Raynolds, M.K.; Comiso, J.C.; Walker, D.A.; Verbyla, D. Relationship between satellite-derived land surface temperatures, arctic vegetation types, and NDVI. Remote Sens. Environ. 2008, 112, 1884-1894.

34. Jiang, Z.; Huete, A.R.; Chen, J.; Chen, Y.; Li, J.; Yan, G.; Zhang, X. Analysis of NDVI and scaled difference vegetation index retrievals of vegetation fraction. Remote Sens. Environ. 2006, 101, 366-378.

35. Gutman, G.; Ignatov, A. The derivation of the green vegetation fraction from NOAA/AVHRR data for use in numerical weather prediction models. Int. J. Remote Sens. 1998, 8, 1533-1543.

36. Chen, X.; Wang, H. Spatial and temporal variations in vegetation zone in Inner Mongolia and vegetation coverage from 1982 to 2003. Scientia Geographica Sinica 2009, 1, 84-94. (In Chinese)

37. Ma, Z.; Shen, T.; Zhang, J.; Li, C. Analysis of vegetation coverage. Bull. Surv. Mapp. 2007, 3, 45-48. (In Chinese)

38. Rong, Y.; Lu, J.; Duan, B.; Yang, Z. Research on urbanization and farmland protection in Henan province based on farmland pressure index. J. Henan Agric. Univ. 2014, 2, 219-223. (In Chinese)

39. Fang, J.; Chen, A.; Peng, C.; Zhao, S.; Ci, L. Changes in Forest Biomass Carbon Storage in China between 1949 and 1998. Science 2001, 292, 2320-2322.

40. Ren, J.; Chen, L. Landform Characters Analysis and Classifying of Henan Province Based on Digital Elevation Model in Shuttle Radar Topography Mission Data Format. Henan Sci. 2011, 9 , 1113-1116. (In Chinese)

(C) 2015 by the authors; licensee MDPI, Basel, Switzerland. This article is an open access article distributed under the terms and conditions of the Creative Commons Attribution license (http://creativecommons.org/licenses/by/4.0/). 